\title{
Effects of AV delay programming on ventricular resynchronisation: role of radionuclide ventriculography
}

\author{
Patrick T. Siegrist $\cdot$ Nathalie Comte $\cdot$ \\ Johannes Holzmeister • Gabor Sütsch • Pascal Koepfli • \\ Mehdi Namdar • Firat Duru • Corinna Brunckhorst • \\ Christoph Scharf • Philipp A. Kaufmann
}

Received: 22 June 2007 / Accepted: 15 February 2008 / Published online: 19 March 2008

(C) Springer-Verlag 2008

\begin{abstract}
Purpose Optimal atrioventricular delay (AVD) setting for cardiac resynchronisation therapy, i.e. biventricular pacing in patients with heart failure, remains a formidable challenge. Thus, the purpose of this study was to evaluate the effects of different AVD on inter- and intra-ventricular resynchronisation using phase histograms of radionuclide ventriculography (RNV).

Methods In 17 consecutive patients (mean age $64 \pm 6$ years), RNV was performed $236 \pm 350$ days after pacemaker implantation for cardiac resynchronisation therapy. Images were acquired during atrial pacing at $80 \mathrm{bpm}$ and during biventricular pacing with AVD ranging from 80 to $160 \mathrm{~ms}$. Inter-ventricular dyssynchrony was measured by the delay between the mean phase angles of the left and right ventricles. Intra-ventricular dyssynchrony was measured by the standard deviation (SD) of left ventricular phase histograms.

Results Left ventricular (LV) ejection fraction (EF) was inversely correlated to LV dyssynchrony (SD of LV phase histogram, $R=-0.82, p<0.0001)$. However, the increase in LVEF by biventricular pacing (mean $+4.4 \pm 4 \%$ ) showed only modest correlation to the resulting resynchronisation
\end{abstract}

Christoph Scharf and Philipp A. Kaufmann contributed equally to the study.

P. T. Siegrist $\cdot$ N. Comte $\cdot$ J. Holzmeister $\cdot$ G. Sütsch $\cdot$ P. Koepfli $\cdot$

M. Namdar $\cdot$ F. Duru $\cdot$ C. Brunckhorst $\cdot$ C. Scharf

P. A. Kaufmann $(\square)$

Cardiovascular Center, Universitiy Hospital Zurich NUK C 32,

Raemistrasse 100,

$\mathrm{CH}-8091$ Zurich, Switzerland

e-mail: pak@usz.ch

P. A. Kaufmann

Zurich Center for Integrative Human Physiology,

University of Zurich,

Zurich, Switzerland effect (characterised by a $-13 \pm 8^{\circ}$ decrease in LV phase histogram SD, $R=-0.38, p<0.0001$ ).

Conclusion RNV is helpful in optimising pacing parameters for resynchronisation therapy. Varying AVD did not have a major impact on intra- or inter-ventricular resynchronisation. Thus, the benefit of AVD-based LVEF optimisation seems to result from atrioventricular resynchronisation.

Keywords Cardiology ventricular function · Imaging ·

Radionucluide ventriculography .

Cardiac resynchronisation therapy

\section{Introduction}

In chronic congestive heart failure, dyssynchrony of left versus right ventricle (RV) as well as atrioventricular (AV) dyssynchrony add to progressive aggravation of the cardiac performance. In addition to optimised medical therapy, biventricular pacing devices have been shown to improve left ventricular (LV) function and prognosis by resynchronising LV and RV contraction [1]. However, the so-called cardiac resynchronisation therapy (CRT) not only acts via inter-ventricular and intra-ventricular resynchronizations [2, 3] but also allows to optimise the timing of atrial and ventricular systoles, i.e. AV resynchronisation $[4,5]$. It has recently been shown that the LV dyssynchrony as assessed with gated single photon emission computed tomography (SPECT) may be used to predict response to CRT [6]. SPECT, however, may provide only limited information, as it only allows to assess the LV function, i.e. intraventricular dyssynchrony. By contrast, radionuclide ventriculography (RNV) has been successfully used not only to quantify LV, but also inter-ventricular dyssynchrony by analysis of phase histograms and their standard deviation (SD) in dilated cardiomyopathy $[7,8]$ as well as to assess 
the effects of biventricular pacing [9]. Although comprehensive CRT includes AV resynchronisation by left atrial pacing and RNV offers the unique opportunity (magnetic resonance imaging is not an option in patients with implanted devices) to assess the effects of different atrioventricular delays (AVDs) on inter- and intra-ventricular resynchronisations, this has not been assessed so far. Averaging a high number of cardiac cycles during AVD optimisation has the advantage of equalising the respiratory changes in contrast to the conventionally used beat-to-beat analysis of the echocardiographic [3] or haemodynamic response [10]. In addition, the global performance of contraction can be assessed, and fully automated image acquisition and calculation minimise operator bias. The purpose of this study was to assess the effect of AVD optimisation on inter- and intra-ventricular resynchronisations using phase images of RNV.

\section{Methods}

\section{Patients}

The subjects of this study were 17 consecutive patients undergoing CRT optimisation after a mean of $236 \pm 350$ days post-implantation at our institution. Pre-implantation data were collected retrospectively and are summarised in Table 1. All patients had symptomatic congestive heart failure (New York Heart Association Classification $\geq 2$ ) and LV ejection fraction $(\mathrm{EF})<35 \%$ associated with one of the

Table 1 Patient characteristics

\begin{tabular}{ll}
\hline Patients & $n=17$ \\
\hline Age (yrs, mean \pm SD) & $64 \pm 6$ \\
Ischemic cardiomyopathy & $10(59)$ \\
Valvular heart disease & $5(29)$ \\
moderate to severe MR untreated & 3 \\
mechanical aortic valve replacement & 1 \\
mechanical aortic and mitral valve replacement & 1 \\
Nonischemic cardiomyopathy & 2 \\
LVEF (\%, baseline echocardiography) & $21(7)$, range 11 \\
& to 35 \\
EDVI (ml, baseline echo) & $188 \pm 43$ \\
End-diastolic diameter (mm, baseline echo) & $80 \pm 6$ \\
QRS LBBB (ms, $n=13)$ & $177 \pm 30$ \\
RBBB, LAHB + AVB1 (ms, $n=1)$ & 135 \\
PQ interval (ms) & $213 \pm 45$ \\
Severe AVB first degree ( $>250$ ms) & 2 \\
Pacemaker dependent & 3 \\
\hline
\end{tabular}

Values are presented as $n(\%)$ unless otherwise indicated. $L V E F$ left ventricular ejection fraction, $E D V I$ end-diastolic volume index, $\angle B B B$ left bundle branch block, $R B B B$ right bundle branch block, AVB1 AV block first degree following indications for CRT: complete AV block (AVB), complete left bundle branch block (LBBB) with $\mathrm{QRS} \geq$ $150 \mathrm{~ms}$ or AVB first degree $\geq 220 \mathrm{~ms}$ associated with a QRS $>$ $120 \mathrm{~ms}$ either due to LBBB or bifascicular block (right bundle branch block+left anterior hemiblock in one patient). The RV lead had been implanted in the RV apex, the right atrial lead in the inter-atrial septum or in the right atrial appendage if necessary for sensing or stability reasons, and the LV lead was implanted into the posterolateral coronary sinus branch at the position with the latest electrical activation during RV pacing, after sampling at least three positions within more than one coronary sinus branch.

\section{Pacing protocol}

To eliminate the effects of heart rate on resynchronisation, all patients were studied at paced rates of $80 \mathrm{bpm}$. Baseline images were obtained during intrinsic conduction in 12 patients and with RV pacing only at AVD $180 \mathrm{~ms}$ in three patients with complete AVB and in 2 patients with severe AVB first degree. Then, biventricular pacing was performed without inter-ventricular delay at different AVD (80, 100, 120,140 and $160 \mathrm{~ms}$ ) in random order to assess the best AVD for each individual patient. All patients had stable sinus rhythm with extrasystoles less than $2 \%$ according to the acquisition reports.

\section{Radionuclide ventriculography}

Erythrocyte labeling was performed using $1,000 \mathrm{MBq}{ }^{99 \mathrm{~m}} \mathrm{Tc}$ pertechnetate $30 \mathrm{~min}$ after an intravenous bolus of $11.9 \mathrm{mg}$ pyrophosphate solved in $10 \mathrm{ml}$ saline. All studies were acquired for 10-12 min with 64 frames per RR interval in the "best septal" left anterior oblique position using a Siemens Diacam scintigraphic scanner (Siemens AG, Erlangen, Germany). The electrocardiogram was monitored continuously to ensure R-wave gating of the QRS complex. Scintigrams were acquired for each patient in sinus rhythm and during biventricular pacing for each AVD. For a processing purpose, raw scintigraphic data were transformed to an ENTEGRA workstation (GE Medical Systems, Milwaukee, WI, USA). LV regions of interest were drawn manually at end-diastole and end-systole by two independent observers unaware of the study condition for calculation of inter-observer variability. After correction for background counts, LVEF was calculated by dividing the difference of end-diastolic counts (EDC) and end-systolic counts (ESC) by EDC:

$\mathrm{LVEF}=(\mathrm{EDC}-\mathrm{ESC}) / \mathrm{EDC}$

Phase images were generated from the scintigraphic data using the PMOD software package (PMOD Technologies, http://www.pmod.com). As the time activity curve and the 
ventricular volume curve have a configuration that approximates a cosine function, the first harmonic cosine term alone is a reasonable approximation. Thus, the time-activity curve of each LV pixel can be described by its phase and amplitude, in the form:

$f(t)=A \cos (t+c)$

where $f(t)$ represents the activity of any pixel as a function of time after the R-wave, $A$ the amplitude of the fitted function, which is an index of how dynamically that pixel is contracting, $c$ represents the phase shift (given as angle) of the cosine function, which is an index of the time in the cardiac cycle (total of $360^{\circ}$ ) when the pixel contracts relative to the R-wave.

The phase program computed the phase and amplitude of the first Fourier harmonic of the time-activity curve of each pixel in the field of view. The color-encoded phase image graphically demonstrates each motion phase of every $\mathrm{LV}$ region using a continuous rainbow color wheel, corresponding to phase angles from one R-wave $\left(0^{\circ}\right)$ to the following R-wave $\left(360^{\circ}\right)$. Thus, one cardiac cycle represents $360^{\circ}$.

Mean phase angles were computed for RV and LV blood pools as arithmetic mean phase angle for all pixels in the ventricular region of interest. The difference between LV and RV mean phase angle was used to calculate interventricular dyssynchrony (LV-RV delay). Intra-ventricular contractile synchrony was measured using the SD of the mean phase angle of the LV blood pool (Fig. 1).

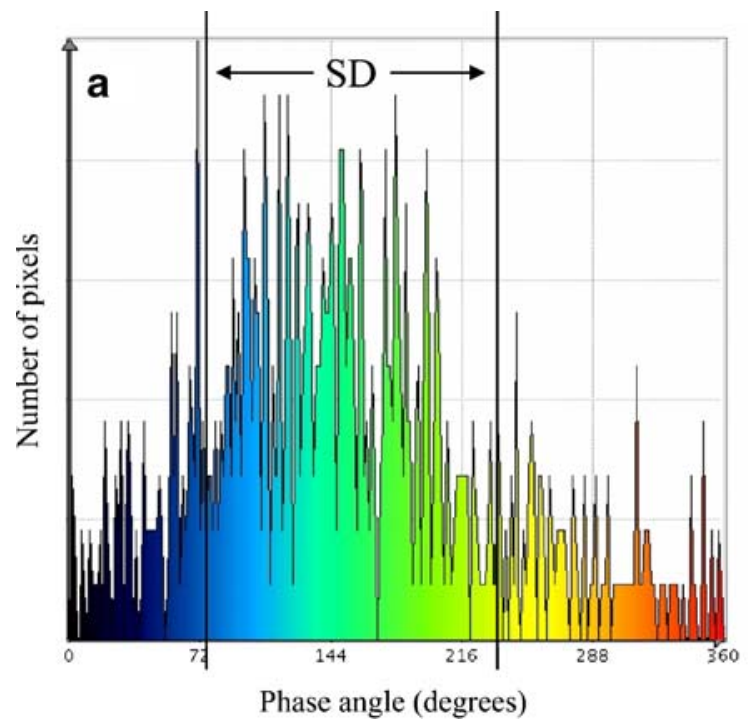

Fig. 1 Effect of CRT on intra-ventricular resynchronisation: phase histograms of the left ventricle illustrating dispersion of phase angles during ventricular ejection, plotted as phase angle ( $x$-axis) versus number of pixels ( $y$-axis). With CRT off, a there is large dispersion of
Statistical analysis

Continuous variables are expressed as mean $\pm \mathrm{SD}$ and are compared using Student's $t$ test. Correlations are expressed using Pearson's coefficients. Inter-observer variability for continuous measurements was assessed according to Bland and Altman. Comparison of repeat measurements was performed with analysis of variance statistic for repeat measurements. A statistical significance was assumed at $p<0.05$, and calculations were performed using a commercially available software package (SPSS 13.0 for Windows).

\section{Results}

In 17 patients with recent biventricular pacemaker implants, six series of images were obtained during baseline rhythm, i.e. with biventricular CRT pacing switched off (CRT off) and biventricular pacing switched on (CRT on) adding up to a total of 102 series. A time frame of $11.7 \mathrm{~ms}$ (64 frames per $750 \mathrm{~ms}$ ) was obtained in all patients from which EF and phase histograms were calculated. LVEF values of the two observers showed an excellent correlation $(R=0.941$, $p<0.0001, \mathrm{SEE}=0.54)$ with narrow Bland-Altman limits of agreement $(-4.9 \%$ to $6.9 \%)$.

Biventricular pacing acutely improved EF by $4.4 \pm 4 \%$ (range $0-15 \%$ ) compared to CRT off. The best AVD was $135 \pm 40 \mathrm{~ms}$, but showed a wide range from $80 \mathrm{~ms}$ AVD to intrinsic rhythm in one patient.

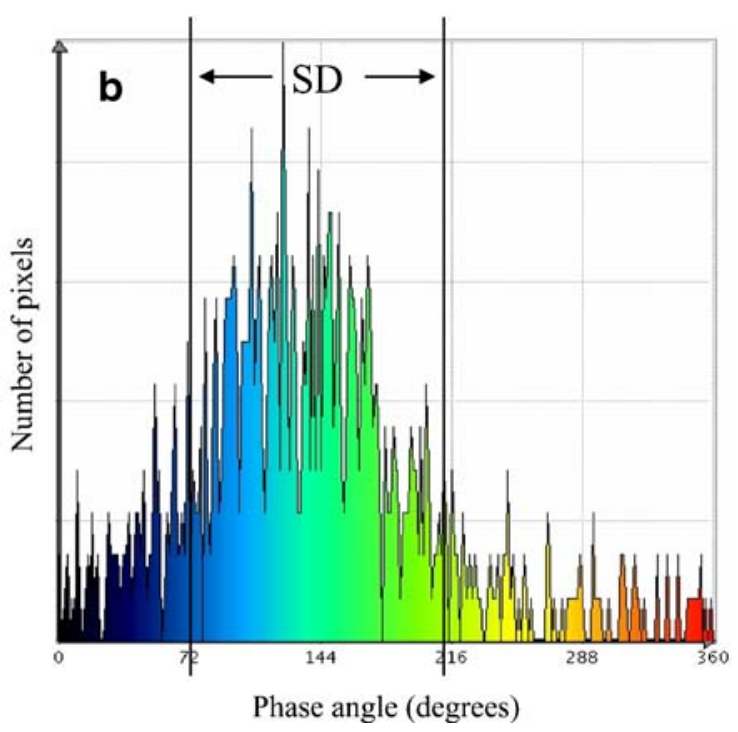

the phase angle $\left(155 \pm 78^{\circ}\right)$ indicating LV dyssynchrony, while CRT b induces a substantial narrowing of the angle $\left(141 \pm 68^{\circ}\right)$ indicating effective resynchronisation. Both measurements were done in the same patient at a heart rate of $80 \mathrm{bpm}$ 
Relation of dyssynchrony to LV function and CRT effects

During RNV, the LVEF with CRT off correlated with baseline LV dyssynchrony expressed as the SD of the LV phase histogram $(R=-0.82, p<0.001)$. This correlation was maintained when data from all six different AVD in each of the 17 patients (i.e. 102 measurements) were included $(R=-0.82, p<0.001$; Fig. 2). By contrast, the relation of LVEF to the inter-ventricular dyssynchrony (difference between mean LV and RV phase angle) was poor $(R=0.27$, $p<0.01$ ) and to the RV intra-ventricular dyssynchrony (SD of $\mathrm{RV}$ phase histogram) was lacking $(R=0.13, p=0.2)$. At optimised AVD, the LVEF improved by a mean of $4.4 \pm 4 \%$ (range $0-15 \%, p<0.001$ ), and the SD of the LV phase histogram decreased by a mean of $13 \pm 8^{\circ}$ (range $3-31^{\circ}$, $p<0.001$ ). Although the improvement of LVEF was not related to the baseline LV dyssynchrony (SD of LV histograms, $R=0.02, p=0.95$ ), there was a modest but significant correlation with the improvement in resynchronisation of the LV (decrease in SD of LV phase histograms, $R=0.38$, $p<0.0001)$. Changes in inter-ventricular dyssynchrony (RV-LV phase angle) or RV SD were not correlated to improvement in LVEF.

\section{Relation of dyssynchrony to AVD programming}

Mean baseline intra-ventricular LV dyssynchrony (SD of phase histogram) was significantly higher than during CRT at any AVD (Table 2, $p<0.001$ ). Furthermore, when analysing individual patients, the same pattern was observed: Baseline LV dyssynchrony improved by any AVD and changes in LVSD among different AVD were minor compared with the improvement from baseline (Fig. 3). This

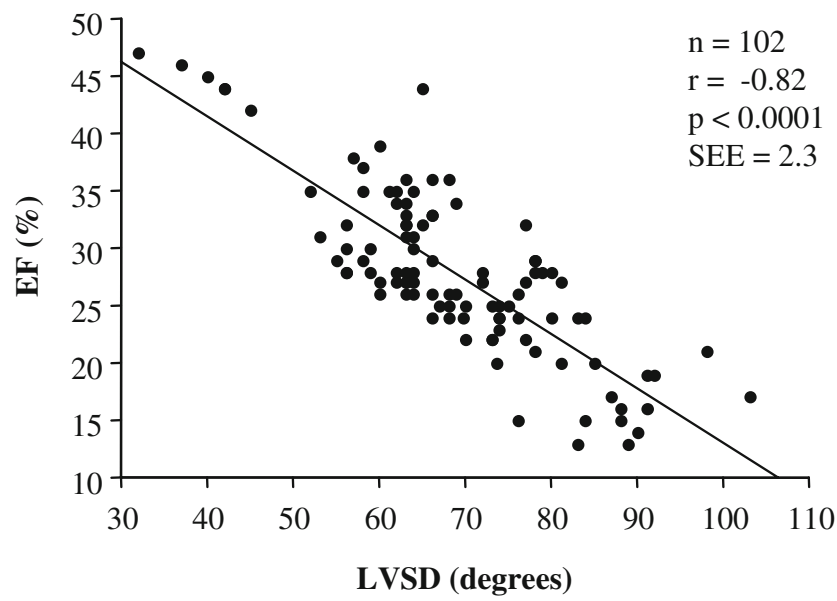

Fig. 2 There was a strong correlation between decreasing LV ejection fraction $(E F)$ and progressive LV dyssynchrony, indicated by a large dispersion of the SD of the LV phase angle (LVSD). Under chronic biventricular stimulation $\mathrm{EF}$ had improved $<35 \%$ in three CRT responders effect was similar in patients with intact $\mathrm{AV}$ conduction compared to patients with AVB.

The mean baseline inter-ventricular dyssynchrony also decreased significantly during CRT at any AVD (Table 2); however, when looking at individual patients, this effect was not consistent (Fig. 3). In six patients (Fig. 3), the interventricular delay during CRT worsened, even though the intra-ventricular synchrony and LVEF improved by CRT. Resynchronisation of the right ventricle (SD of RV) was not observed during CRT compared to baseline values (Table 2).

\section{Discussion}

This study is the first to elucidate the effects of AVD programming on ventricular resynchronisation with the use of RNV. First, we confirmed the current understanding of resynchronisation: LV dyssynchrony (SD of phase histograms) is related to the degree of contractile dysfunction (EF), while resynchronisation (characterised by a decrease in SD of LV phase histograms, Fig. 1) is related to the acute improvement in LVEF. The second finding of this study has important clinical implications: Although CRT led to an improvement in LV resynchronisation (decrease in LVSD) in all patients, this improvement was independent from the respective AVD. Thus, any AVD between 80 and $160 \mathrm{~ms}$, as long as biventricular capture was preserved, led to a similar degree of improvement in mean LV resynchronisation, and this effect was also observed in patients with preserved AV conduction (Fig. 3). In fact, inter-ventricular dyssynchrony was not affected by AVD optimisation in a distinctive pattern. Therefore, the effects of AVD optimisation on LVEF, being independent of inter- or intra-ventricular resynchronisation, appear to result from individually optimised $A V$ synchronisation.

This finding elucidates an important mechanism of resynchronisation: As long as biventricular capture is preserved, the intrinsic electrical activation within the HisPurkinje system has no major impact on timing of ventricular systolic contraction. The global activation imaging provided by RNV, which is averaged over a large number of cardiac cycles, is an excellent tool to evaluate any impact of AVD programming on ventricular resynchronisation.

Major effects of AVD optimisation on cardiac performance in conventional [11] and biventricular pacing [12] are known in decades. However, the effect of different AVD programming on intra- and inter-ventricular resynchronisations has not been elucidated. Our study suggests indirectly that the benefit of AVD optimisation results from improved timing of atrial systole. This hypothesis, namely the fact that AV resynchronisation plays an important role in CRT, is in line with recent studies comparing different pacing modes (i.e. 
Table 2 Results of AVD optimisation on EF, inter- and intra-ventricular synchronisations

\begin{tabular}{lccccrrr}
\hline & CRT off & AVD 80 & AVD 100 & AVD 120 & AVD 140 & AVD 160 & Indiv. AVD at best EF \\
\hline EF (\%) & $26.4 \pm 6.5$ & $28.1 \pm 7.6$ & $27.4 \pm 7.8$ & $28.5 \pm 8.5$ & $28.0 \pm 7.3$ & $28.5 \pm 7.3$ & $30.5 \pm 7.4 *$ \\
Inter-D & $14.8 \pm 19.8$ & $10.5 \pm 25.6$ & $9 \pm 27.2$ & $10.8 \pm 27.5$ & $9.2 \pm 25.3$ & $8.7 \pm 25.0$ & $10.4 \pm 24.9$ \\
LV avr & $177.8 \pm 18.7$ & $173 \pm 20$ & $176 \pm 20$ & $173.3 \pm 14.6$ & $174.8 \pm 14.6$ & $171.5 \pm 16.3$ & $172.5 \pm 17.9$ \\
LVSD & $76.0 \pm 14.5$ & $66.1 \pm 13$ & $66.5 \pm 13$ & $67.2 \pm 11.8$ & $68.4 \pm 11.5$ & $67.6 \pm 11.2$ & $66.2 \pm 13.8 *$ \\
RV avr & $163.0 \pm 17.9$ & $163.0 \pm 20$ & $167 \pm 22$ & $162.5 \pm 18.6$ & $165.6 \pm 19.4$ & $162.8 \pm 16.6$ & $162.1 \pm 16.8$ \\
RV SD & $57.5 \pm 16.2$ & $53.6 \pm 18$ & $56.8 \pm 15$ & $55.6 \pm 14.3$ & $56.9 \pm 15.5$ & $60.2 \pm 14.4$ & $55.3 \pm 15.9$ \\
\hline
\end{tabular}

Data are mean \pm SD.

$E F$ ejection fraction, Inter- $D$ inter-ventricular delay (difference mean phase angles RV-LV), $L V$ avr average LV phase angle, $R V$ avr average RV phase angle, LVSD SD of left ventricle phase histograms, RVSD SD of RV phase histograms, CRT off cardiac resynchronisation therapy, $A V D$ atrioventricular delay

${ }^{*} \mathrm{p}<0.01$ vs CRT off

a

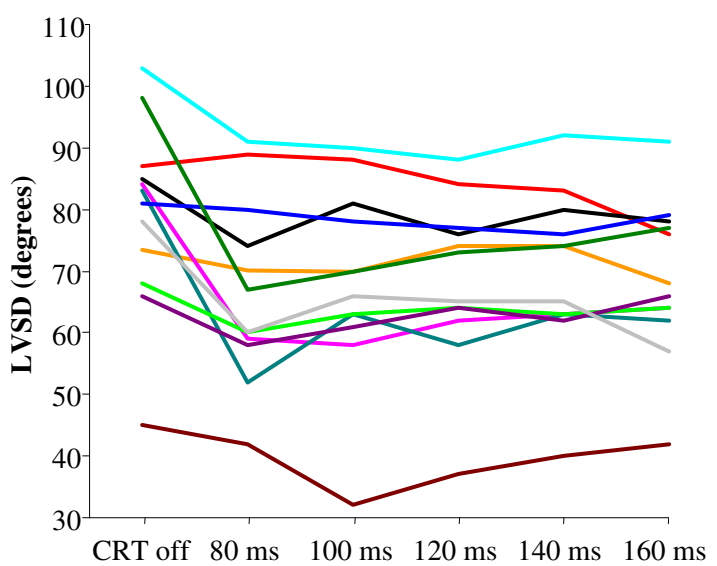

C

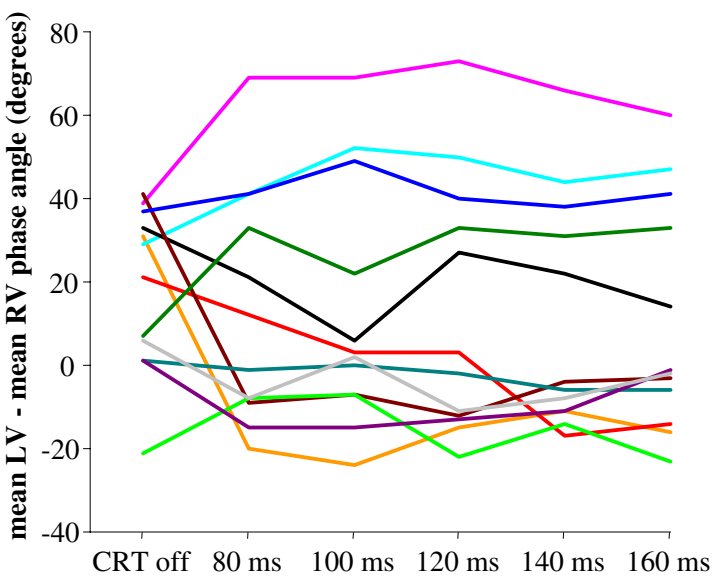

Fig. 3 Effect of different AVD on intra-ventricular resynchronisation in each patient: SD of mean phase angle in patients without (a) and with AVB (b). Effects of different AVD on inter-ventricular resynchronisation: difference of mean phase angle between right and left ventricle in patients without (c) and with AVB (d). Baseline

\section{b}

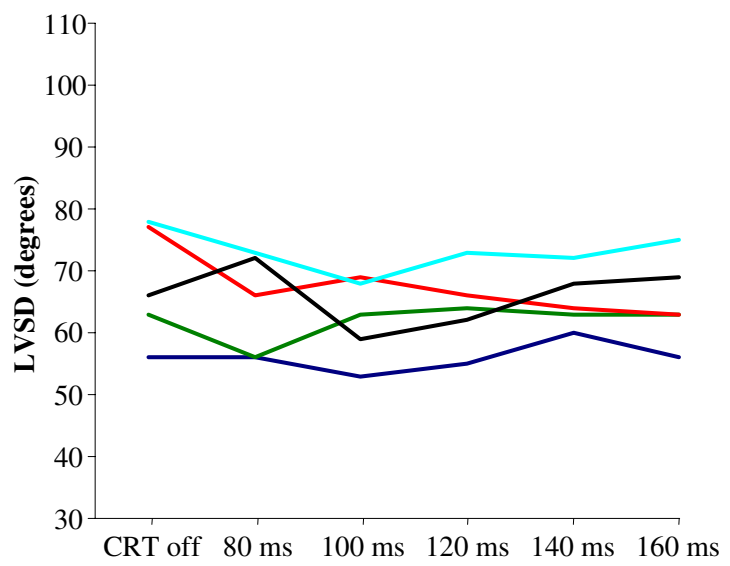

d

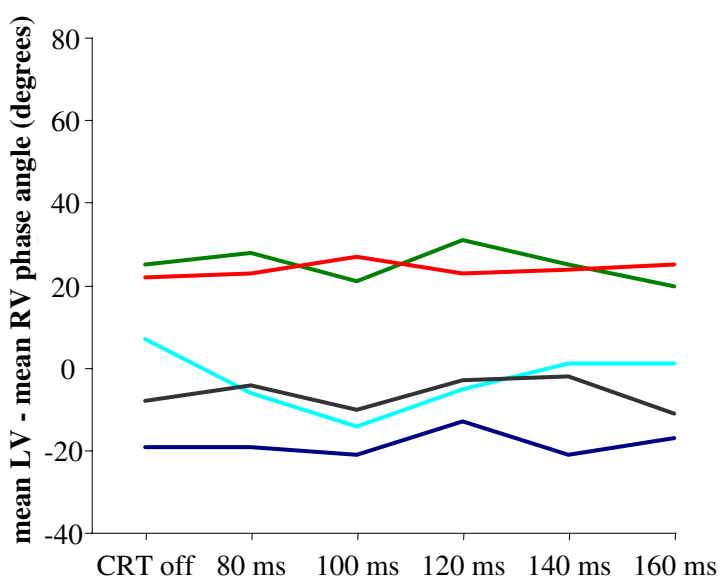

images (CRT off) were obtained during atrial pacing with $80 \mathrm{~ms}$ AVD in patients without $\mathrm{AVB}$ and during right ventricular pacing with $180 \mathrm{~ms}$ AVD in patients with significant AVB (PQ intervals $>250 \mathrm{~ms}$ or AVB third degree). $R V$ right ventricular, $L V$ left ventricular, $C R T$ cardiac resynchronisation therapy, $S D$ standard deviation 
sensing only in the ventricle or sensing and pacing in all chambers) [13] and analysing PR intervals when performing inter-ventricular and AVD optimisations [14].

RNV for measurement of dyssynchrony

and resynchronisation

Reproducibility of LVEF and cardiac dyssynchrony in heart failure remains challenging. Sampling a high number of cardiac beats during image acquisition in RNV equalises beat-to-beat variability and the effects of respiration. By using automatic image acquisition and analysis, the measurements are less operator dependent than with echocardiography. The quantification of inter- and intraventricular dyssynchrony by comparing SD of phase histograms has been successfully demonstrated and proven to be of prognostic relevance [8]. Finally, RNV integrates the entire cardiac motion, which is the ultimate goal of the latest echocardiographic techniques as well [15].

\section{Study limitations}

A major limitation of $\mathrm{RNV}$ is that mitral regurgitation is not taken into account and improved LVEF, therefore, does not necessarily translate into a better forward flow. This might explain some discrepancies between our findings and those from echocardiographic studies: In the present study, the magnitude of baseline LV dyssynchrony did not predict acute changes in LVEF, and the best LVEF was observed in a wide range of AVD including baseline rhythm in one patient. However, we used RNV to measure global ventricular resynchronisation reliably and reproducibly during AVD optimisation. Another limitation might be that true electrical fusion between intrinsic conduction and biventricular pacing was not studied. The absence of significant changes in CRT performance with AVD up to $160 \mathrm{~ms}$, which was significantly shorter than the average baseline AV conduction, argues against a significant effect of electrical fusion. In daily clinical routine, AVDs are usually programmed below $160 \mathrm{~ms}$.

\section{Conclusion}

RNV is helpful in optimising pacing parameters for CRT and provides important pathophysiologic information. Varying AVD did not have a major impact on intra- or inter-ventricular resynchronisations. Thus, the benefit of AVD-based LVEF optimisation seems to result from atrioventricular resynchronisation.

Acknowledgements Philipp A. Kaufmann was supported by a grant from the Swiss National Science Foundation (SNSF-professorship grant No. PP00A-114706). Christoph Scharf has received research grants from Medtronic and Guidant and is a consultant to Biotronik. The authors have had full control of all primary data, and we agree to allow the European Journal of Nuclear Medicine and Molecular Imaging to review such data if requested.

\section{References}

1. Cleland JGF, Daubert JC, Erdmann E, Freemantle N, Gras D, Kappenberger L, et al. The effect of cardiac resynchronization on morbidity and mortality in heart failure. NEJM 2005;352:153949.

2. Cazeau S, Jauvert G, Alonso C, Casset C, Ranaivoson H, Cazeau $\mathrm{S}$, et al. Echocardiographic modelling of cardiac asynchronism: prospective evaluation before and after multisite stimulation. Arch Mal Coeur Vaiss 2003;96:659-64.

3. Breithardt OA, Stellbrink C, Franke A, Balta O, Diem HB, Bakker $\mathrm{P}$, et al. Acute effects of cardiac resynchronisation therapy on left ventricular Doppler indices in patients with congestive heart failure. Am Heart J 2002;143:34-44.

4. Auricchio A, Ding J, Spinelli JC, Ding J, Pochet T, Vernooy K, et al. Cardiac resynchronisation therapy restores optimal atrioventricular mechanical timing in heart failure patients with ventricular conduction delay. J Am Coll Cardiol 2002;39:1163-9.

5. Ritter P, Dib JC, Mahaux V, Levièure T, Soyeur D, Lavargne T, et al. New method for determining the optimal atrio-ventricular delay in patients paced in DDD mode for complete atrioventricular block. PACE 1995 1995;18(Suppl II):855.

6. Henneman MM, Chen J, Dibbets-Schneider P, Stokkel MP, Bleeker GB, Ypenburg C, et al. Can LV dyssynchrony as assessed with phase analysis on gated myocardial perfusion SPECT predict response to CRT? JNM 2007;48(7):1104-11.

7. Fauchier L, Marie O, Casset-Senon D, Babuty D, Cosnay P, Fauchier JP. Interventricular and intraventricular dyssynchrony in idiopathic dilated cardiomyopathy: a prognostic study with fourier phase analysis of radionuclide angioscintigraphy. J Am Coll Cardiol 2002;40:2022-30.

8. Bader H, Garrigue S, Lafitte S, Reuter S, Jais P, Haissaguerre M, et al. Intra-left ventricular electromechanical asynchrony. A new independent predictor of severe cardiac events in heart failure patients. J Am Coll Cardiol 2004;43:248-56.

9. Kerwin WF, Botvinick EH, O'Connell JW, Merrick SH, DeMarco $\mathrm{T}$, Chatterjee $\mathrm{K}$, et al. Ventricular contraction abnormalities in dilated cardiomyopathy: effect of biventricular pacing to correct interventricular dyssynchrony. J Am Coll Cardiol 2000;35:12217.

10. Stellbrink C, Breithardt OA, Franke A, Sack S, Bakker P, Aurichio A, et al. Impact of cardiac resynchronisation therapy using haemodynamically optimised pacing on left ventricular remodeling in patients with congestive heart failure and ventricular conduction disturbances. J Am Coll Cardiol 2001;38:195765.

11. Hochleitner M, Hortnagl H, Ng CK, Hortnagel H, Gschnitzer F, Zechmann W. Usefulness of physiologic dual-chamber pacing in drug-resistant idiopathic dilated cardiomyopathy. Am J Cardiol 1990;66:198-202.

12. Auricchio A, Stellbrink C, Block M, Sack S, Vogt J, Bakker P, et al. Effect of pacing chamber and atrioventricular delay on acute systolic function of paced patients with congestive heart failure. The Pacing Therapies for Congestive Heart Failure Study Group. The Guidant Congestive Heart Failure Research Group. Circulation 1999;99:2993-3001. 
13. Bernheim A, Ammann P, Sticherling C, Burger P, Schaer B, Brunner-La Rocca HP, et al. Right atrial pacing impairs cardiac function during resynchronisation therapy: acute effects of DDD pacing compared to VDD pacing. J Am Coll Cardiol 2005;45:1482-7.

14. Vanderheyden M, De Backer T, Rivero-Ayerza M, Geelen P, Bartunek J, Verstreken S, et al. Tailored echocardiographic interventricular delay programming further optimizes left ventricular performance after cardiac resynchronisation therapy. Heart Rhythm 2005;2:1066-72.

15. Kapetanakis S, Kearney MT, Siva A, Gall N, Cooklin M, Monaghan MJ. Real-time three-dimensional echocardiography: a novel technique to quantify global left ventricular mechanical dyssynchrony. Circulation 2005;112:992-1000. 